Código JEL: M53, M54, J24

\title{
La formación en los procesos de generación de capital humano
}

\author{
Javier ALONSO LÓPEZ \\ Dpto. Organización de Empresas \\ Universidad Complutense de Madrid \\ alonsolopez.javier@gmail.com \\ Ignacio DANVILA DEL VALLE \\ Profesor Ayudante Doctor del Dpto. Organización de Empresas \\ Universidad Complutense de Madrid \\ idanvila@ccee.ucm.es
}

Recibido: 26-04-2011

Aceptado: 17-11-2011

\section{RESUMEN}

Las organizaciones de hoy en día deben responder a las demandas del entorno y en consecuencia deben saber adaptarse a las mismas. El directivo debe poseer no sólo conocimientos de índole económicoadministrativo, sino que debe reunir características personales y demostrar habilidades directivas y comunicativas en el ejercicio de su puesto. Por este motivo, las organizaciones precisan directivos con una adecuada formación. Dicha formación puede influir directamente en los resultados obtenidos por estas instituciones. En este artículo se realiza una revisión de este concepto y se esbozan líneas de investigación relacionadas con este tema.

Palabras clave: Recursos Humanos; Formación; Capital Humano; rentabilidad. 


\title{
Training in the generation of human capital
}

\begin{abstract}
Nowadays, the organizations must meet the demands of the environment and therefore should be able to adapt to them. The manager must possess not only knowledge of economic and administrative nature, but must gather personal characteristics and demonstrate leadership skills and communicative in his tenure. For this reason, organizations need managers with adequate training. Such training can directly influence the results obtained by these institutions. In this article we review this concept and outlines areas of research related to this topic.
\end{abstract}

Keywords: Human Resource; Trining; Human Capital; Profitability.

Sumario: 1. Introducción. 2. El capital humano en el marco de los recursos y capacidades de la empresa. 3. Los procesos de formación en la empresa. 4. La formación y la transferencia de conocimiento. 5. Conclusiones. Bibliografía.

\section{INTRODUCCIÓN}

La razón y los motivos del éxito de las empresas son continuamente estudiados por académicos y profesionales del mundo empresarial. La forma en la que se mide este éxito también ha sido muy analizada. Numerosos estudios utilizan como medida de éxito la rentabilidad de la organización, debido a que los propietarios de las compañías reclaman rendimientos a la inversión, entendida ésta como retorno en forma de beneficios.

Durante la década de los noventa del siglo pasado y hasta la actualidad, se ha producido un cambio en la mentalidad de la dirección de empresas, en lo relativo a la forma de obtener un rendimiento empresarial superior al de la competencia. En este campo adquiere gran relevancia la Teoría de Recursos y Capacidades (Barney, 1991, 1992; Wernefelt, 1984; Dierickx, Cool, y Barney, 1989; Grant, 1996; Rumelt, 1991, Amit y Schoemaker, 1993; Peteraf, 1993; Huselid, 1995; Barney y Wright, 1998) que centra su atención en el ámbito interno de la empresa frente a otros factores como son el entorno o el sector industrial en el que se compite, para obtener rentas superiores a las de la competencia. Esta teoría, entiende la empresa como un conjunto de recursos y capacidades únicas y singulares.

La ventaja competitiva proviene de los recursos y capacidades que posee la organización, buscando fundamentalmente que sean escasos, valiosos, inimitables e insustituibles. Estos recursos y capacidades que la empresa controla, generan una ventaja competitiva, al entenderse como aquellas acciones que la organización hace mejor que sus competidores (Peteraf, 1993). 
El pretende trabajo pretende explicar cómo un programa de formación en dirección empresarial puede influir en la rentabilidad de estas organizaciones. Vamos a analizar si para mejorar las organizaciones, los directivos necesitan, y si ésta formación influye directamente en los resultados obtenidos en dichas instituciones. Concretamente, estudiaremos la influencia de la formación en la rentabilidad y la coherencia entre las necesidades y la formación recibida.

Las organizaciones deben responder a las demandas del entorno y saber adaptarse al mismo. El directivo debe poseer conocimientos económicos, ciertas características personales y demostrar habilidades en el ejercicio de su puesto.

Los directivos no pueden ser sólo un elemento integrador de un grupo, sino que además tienen que ser capaces de administrar de manera rentable la organización. Podemos afirmar que no existen organizaciones buenas con malos directivos y viceversa.

Numerosos trabajos, consideran la formación de los empleados como una de las variables críticas que permite la obtención de la ventaja competitiva, y por tanto la obtención de rentas superiores (Storey, 2004; Danvila y Sastre, 2009; Danvila et al., 2009).

La formación se considera como una de las prácticas de recursos humanos, importante para las empresas y capaz de generar una ventaja competitiva diferenciadora y sostenible en el tiempo.

A pesar de la existencia de numerosos estudios sobre la relación entre formación y resultados, es conveniente seguir investigando en este campo debido a la divergencia en las conclusiones obtenidas.

\section{EL CAPITAL HUMANO EN EL MARCO DE LOS RECURSOS Y CAPA- CIDADES DE LA EMPRESA}

Durante la última década, los Recursos Humanos se han convertido en la fuente principal de las empresas, es decir una de las claves sobre las que se basa el éxito de la organización (Úbeda García, 2005; Van de Wiele, 2010; Stavrou, Brewster y Charalambous, 2010). Cada vez es más frecuente ver en los informes de responsabilidad social corporativa, la importancia que la empresa otorga a los trabajadores, siendo ésta uno de los fundamentos estratégicos.

Con esta nueva visión se han dejado atrás posturas mecanicistas en el tratamiento de la persona, en ambientes de rivalidad entre sectores y dentro de ellos, que producen alienación en la persona. La existencia de las organizaciones, no puede ser considerada sólo como un elemento maximizador de beneficios sin tener en cuenta al recurso humano. Se debe entender la existencia de la empresa como comunidad de hombres que, de diversas maneras, buscan la satisfacción de sus necesidades fundamentales y constituyen un grupo particular al servicio de la sociedad entera.

Los cambios en el tratamiento del concepto de trabajador y de la importancia del capital humano dentro de la estrategia empresarial, han permitido entender el trabajo 
como un bien del hombre, que le permite realizarse a sí mismo. En aras a la consecución de este fin, las condiciones de trabajo han sido modificadas para adaptar la empresa a la persona y no al revés: dicho cambio ha sido facilitado mediante cambios legislativos que han actuado de manera coercitiva.

En una era de máxima rivalidad entre las empresas, adquiere vital importancia el capital humano, por tratarse de un elemento diferenciador. En este sentido, los Recursos Humanos fruto de los conocimientos y capacidades limitadas, proporcionan un hecho diferencial a través del valor que ofrecen, que lo constituyen en Capital Humano.

Los recursos humanos son uno de los recursos internos sobre los que la empresa comienza a planificar su estratégica. A través del capital humano, la organización es capaz de obtener una ventaja competitiva, mediante el desarrollo de habilidades, nuevos conocimientos, cohesión en equipos de trabajo, etc., en definitiva, se busca transformar la capacidad, el conocimiento y la creatividad de las personas en resultados tangibles (De la Rosa y Carmona, 2002). Puede favorecer la creación de una ventaja competitiva difícil de imitar el propio ambiente de trabajo y la cohesión entre sus miembros.

Consideramos a los Recursos Humanos, como uno de los recursos fundamentales sobre los que se puede obtener rentas superiores a las obtenidas por los competidores, sostenibles en el tiempo. Las prácticas de gestión del Capital Humano, de hoy en día, no se entienden como un coste obligatorio, sino como un arma estratégica para la obtención de una ventaja competitiva (Blume et al., 2010).

Consideramos a la empresa como un conjunto de habilidades, conocimientos, tecnología,... que se generan y amplían con el tiempo. De modo que, la estrategia se basa en términos de lo que la empresa sabe o puede llegar a hacer, de su potencial (Barney, 1991).

Partiendo de la concepción anteriormente indicada de empresa y de la importancia del capital humano para el desarrollo de la empresa, consideramos que la Teoría de Recursos y Capacidades es adecuada para fundamentar nuestra investigación. Además, ha sido una de las teorías más utilizadas en la última década en el campo de la Dirección Estratégica. Entendemos que mediante los recursos internos de la organización, se genera valor añadido y por tanto se adquiere una posición relevante en el mercado.

Además de la Teoría de Recursos y Capacidades, existen otros enfoques y teorías que explican la relación e importancia de los recursos humanos para la obtención de una ventaja competitiva sostenible. No obstante, la teoría más utilizada en la literatura académica, es la de recursos y capacidades (Barney, 1991b) y su particular enfoque de la gestión del conocimiento (Bassi et al., 1998) o la teoría del capital humano (Becker, 1983). Desde la Teoría de Recursos y Capacidades, se considera a la empresa como un conjunto único de recursos y capacidades, capaces de generar una ventaja competitiva sostenible, que produzca rentas superiores a las de la competencia. Por tanto podemos considerar que el recurso humano en el que la empresa invertirá tiene el potencial necesario para crear un rendimiento superior (Wright et al., 1994,1998; 
Lado y Wilson, 1994; Kamoche, 1996; Mueller, 1996; Boxall, 1996; Guest, 1997; Barney y Wright, 1998; De Saá y García, 2002).

Desde la perspectiva de la Gestión del Conocimiento (Bassi et al., 1998; Alavi y Leidner, 2001) no sólo es importante el conocimiento individual, sino también el conocimiento colectivo de la organización. En este proceso, la formación tiene un papel fundamental, al poder ser un incentivo para mantener el conocimiento de la empresa, evitando que los trabajadores con conocimientos críticos se marchen a la competencia.

La teoría del Capital Humano (Becker, 1983), introduce la idea de que el desarrollo del factor humano tiene importancia para el crecimiento económico general. Esto supone un apoyo a la explicación del valor que posee la formación, al considerarse una herramienta para el desarrollo humano y la competitividad empresarial. Toda inversión en capital humano, busca la obtención de rendimientos superiores generados a través de aumentos de productividad (Úbeda García, 2005).

Además de las teorías y perspectivas ya señaladas (teoría de recursos y capacidades, enfoque de gestión del conocimiento y teoría del capital humano), existe la Perspectiva Institucional (Rothemberg, 2007) basada en que las empresas adoptan prácticas de recursos humanos (entre ellas la formación), no para adquirir una ventaja competitiva sostenible que suponga en un incremento de la rentabilidad, sino por presiones no competitivas del entorno. Las empresas reflejan en su estructura la realidad social y las presiones no competitivas del entorno, que influyen sobre el comportamiento de las empresas que actúan en él (Meyer y Rowan, 1977; Dimaggio y Powel, 1983), buscando en sus actuaciones la legitimidad, externa e interna. Entendida ésta como el grado en que la estructura, actividades y prácticas de una empresa son coherentes con el contexto económico y social de la misma (Scott, 2001; Dacin et al., 2007).

En nuestro trabajo, pretendemos dar a conocer la ventaja competitiva sostenible obtenida a través del capital humano, en definitiva por medio de recursos y capacidades internas.

La Teoría de Recursos y Capacidades considera a la empresa como un conjunto único de recursos y capacidades, capaces de generar una ventaja competitiva sostenible, que produzca rentas superiores a las de la competencia. Por tanto, sólo a través del desarrollo eficiente de las características internas de la empresa se pueden alcanzar rentas superiores. De esta forma, se rechaza la teoría de la obtención de rentabilidades superiores por la pertenencia a un sector industrial o por otra serie de condicionamientos externos. Si el rendimiento dependiera de características externas a la propia empresa, todas las empresas de un mismo sector deberían obtener beneficios similares.

Entendemos que la Teoría de Recursos y Capacidades no explica en su totalidad la obtención de rentas superiores, sino que debe ser utilizada conjuntamente con otras teorías.

La empresa para ser capaz de obtener una ventaja competitiva, debe poseer recursos heterogéneos, escasos y difíciles de imitar o copiar (Barney, 1991b), y además generar mayor valor para sus clientes que los de la competencia (Ansoff, 1965; De Castro y 
Chrisman, 1995). Por recurso heterogéneo, entendemos la posesión de recursos y capacidades diferentes y por escaso la no disponibilidad para el resto de empresas.

En el análisis de las causas que determinan la competitividad y resultados de la empresa, hemos expuesto cómo la Teoría de Recursos y Capacidades focaliza su atención en el estudio de variables internas, tal y como viene expresado en el cuadro 1.

Diferentes estudios basados en la Teoría de Recursos y Capacidades, establecen que si bien la estrategia empresarial debe fundamentarse en la búsqueda de recursos y capacidades diferenciadores, es necesario un proceso de cambio y aprendizaje continuo que sea capaz de adaptar las fuentes de la ventaja competitiva al contexto dinámico de la industria. Esto se conoce como enfoque de las capacidades dinámicas (Teece, Pisano y Schuen, 1997). Dicho enfoque posee unos elementos que podemos encontrar a través de varios autores: Penrose (1959), Williamson $(1975,1985)$, Nelson y Winter (1982) y Hayes, Wheelwright y Clark (1988).

Cuadro 1: Esquema de la Teoría de Recursos y Capacidades

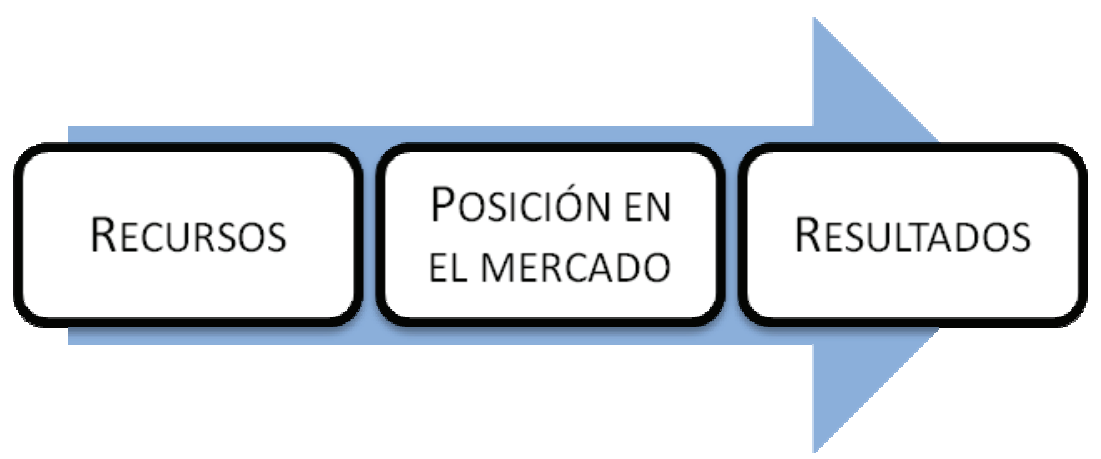

Fuente: Elaboración propia.

Diferentes estudios basados en la Teoría de Recursos y Capacidades, establecen que si bien la estrategia empresarial debe fundamentarse en la búsqueda de recursos y capacidades diferenciadores, es necesario un proceso de cambio y aprendizaje continuo que sea capaz de adaptar las fuentes de la ventaja competitiva al contexto dinámico de la industria. Esto se conoce como enfoque de las capacidades dinámicas (Teece, Pisano y Schuen, 1997). Dicho enfoque posee unos elementos que podemos encontrar a través de varios autores: Penrose (1959), Williamson $(1975,1985)$, Nelson y Winter (1982) y Hayes, Wheelwright y Clark (1988).

La ventaja competitiva de una organización debe ser sostenida en el tiempo, para lo cual los recursos deben poseer unas características (que expondremos más adelante y que son enunciadas por la Teoría de Recursos y Capacidades), pero también tienen que ser capaces de construir y redefinir las competencias para adaptarse a entornos cambiantes (Teece, Pisano y Schuen, 1997). De manera que, que en un primer mo- 
mento la ventaja competitiva se genera sobre unos recursos (enfoque estático) pero posteriormente la empresa debe tener la capacidad de generar nuevas competencias (enfoque dinámico). Por tanto, debe prestar especial atención a los activos que posee (determinantes de la posición que ocupa en el mercado y en el sector así como de la reputación), inversiones realizadas en el pasado (influyen en las capacidades futuras) y proceso de gestión desarrollado por la empresa. Éste podemos considerarlo como el establecimiento de rutinas, aprendizaje y rediseño para adaptarse al entono.

Toda empresa posee una amplia variedad de recursos y capacidades que pone al servicio de la actividad productiva. Estos recursos proporcionan eficiencia organizativa que permite a la empresa obtener una ventaja competitiva que además sea sostenible, de manera que la organización debe conocer cuáles son los recursos con los que cuenta para desarrollar la estrategia empresarial. Para Barney (1986b) son "factores estratégicos" necesarios para implantar la estrategia de la empresa.

Desde la perspectiva de la Teoría de Recursos y Capacidades, los recursos generan valor en tanto en cuanto sean complejos, intangibles y dinámicos. Varios autores consideran que si esto es así, el desarrollo del sistema de Recursos Humanos en la empresa, es especialmente importante como fuente para hacer sostenible una ventaja competitiva (Lado y Wilson, 1994; Pfeffer, 1994; Wright et al., 1994; De Saá y García, 2002).

No obstante, no todos los recursos y capacidades, proporcionan una ventaja competitiva que genera rentas económicas superiores a la competencia y además de modo duradero. La literatura académica ha prestado especial atención al discernimiento y concreción de los rasgos que debe poseer un recurso para obtener la calificación de estratégico. Estos rasgos vienen recogidos en el cuadro 2.

Como hemos indicado, el capital humano posee las condiciones necesarias para generar rentas superiores a las de la competencia. Numerosos estudios analizan la relación entre las políticas de los recursos humanos y los resultados (Bartel, 1994; Black y Lynch, 1996; Klein y Weaver, 2000; Barret y O'Conell, 2001; Aragón et al., 2003; Úbeda García, 2005; Bryan, 2006; Chi, Wu y Lin, 2008; Abdel-Wahab et al., 2008; Stavrou et al., 2010; Van de Wiele, 2010), en dichos estudios aparecen diferentes efectos, destacando una relación positiva entre los dos tópicos. Las prácticas de Recursos Humanos que se pueden encontrar en una empresa son variadas, pero centraremos nuestro trabajo en una de las prácticas de Recursos Humanos. Concretamente la formación y su efecto sobre la rentabilidad empresarial.

Respecto a las diferentes investigaciones que analizan la relación entre formación y rendimientos, destaca Wright y Haggerty (2005). Dicho trabajo muestra la existencia de inconsistencias metodológicas en los estudios que analizan la causalidad entre formación y resultados de la organización. Estas inconsistencias se basan en que la medida del resultado de la empresa no es la misma en todos los trabajo.

El efecto positivo de la formación sobre los resultados empresariales ha sido valorado en distintos contextos culturales, concluyéndose que tal relación es positiva independientemente de la situación cultural que posea el país o región (Fey, y Bjork- 
man, 2001; De Kok, 2002; Ng y Siu, 2004; Úbeda García, 2005; Makó, 2005; Chi, Wu y Lin, 2008).

El efecto positivo de la formación sobre los resultados de la empresa, también ha sido verificado en el contexto de la pequeña y mediana empresa - en adelante PYME , (Patton, Marlow y Hannon, 2000; Ng y Siu, 2004; Storey, 2004; Bacon y Hoque, 2005; Makó, 2005; Chi, Wu y Lin, 2008).

Cuadro 2: Características recursos y capacidades estratégicos

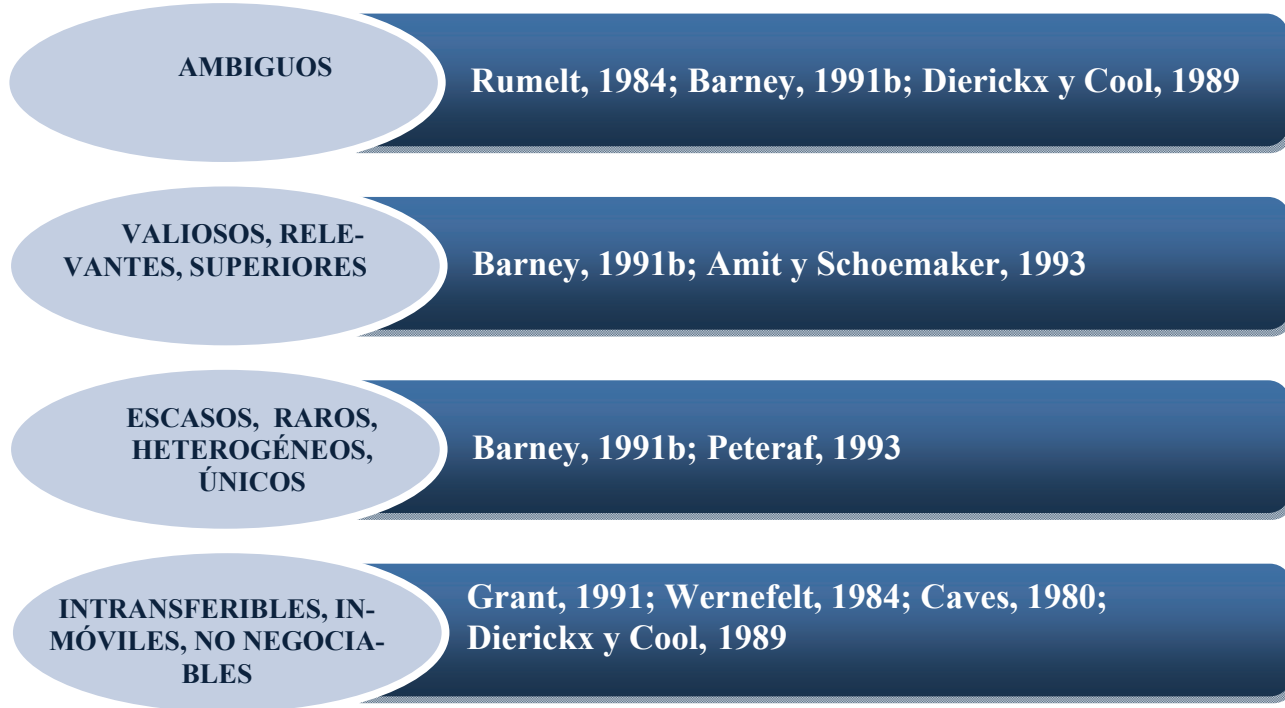

INIMITABLES, MECANISMOS DE AISLAMIENTO, NO TRANSPARENTES, NO REPLICABLES
Dierickx y Cool, 1989; Rumelt, 1991; Barney, 1991b; Grant, 1991; Peteraf, 1993
ESPECÍFICOS

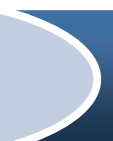

Williamson, 1975; Barney, 1986b; Black y Boal, 1994

\section{COMPLEJOS}

Barney, 1991b; Black y Boal, 1994

INSUSTITUIBLES

Dierickx y Cool, 1989; Barney, 1991b 
Cuadro 2: Características recursos y capacidades estratégicos (cont.)
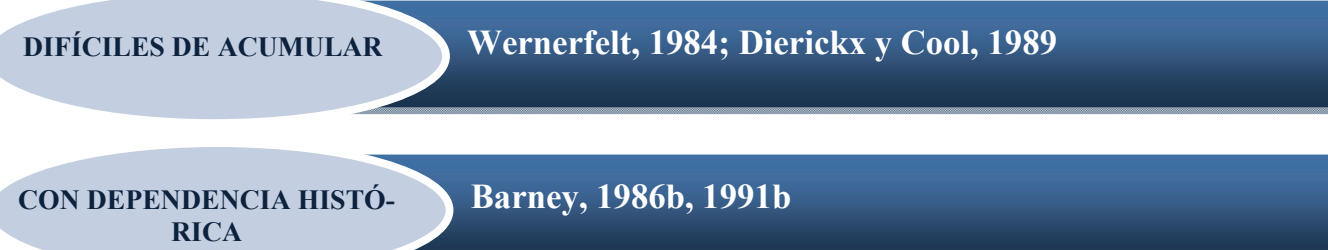

\section{DURADEROS}

APROPIABLES

\section{Ó-} Bar

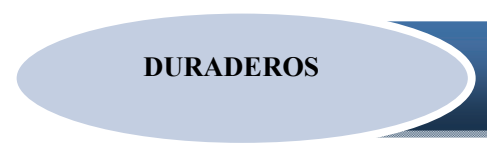

Dierickx y Cool, 1990; Grant, 1991

Fuente: Elaboración propia

Existen estudios en los que se sugiere que la PYME desarrolla las capacidades de sus recursos humanos a través de programas de formación eficaces (Storey, 2004; Beaver y Hutchings, 2005; Úbeda García, 2005; Chi, Wu y Lin, 2008). Buscando en estos programas la generación de capital humano que pueda aumentar el rendimiento de la organización (Lin y Wei, 2005; Chi, Wu y Lin, 2008).

\section{LOS PROCESOS DE FORMACIÓN EN LA EMPRESA}

La formación de los empleados ha adquirido una relevancia capital para el desarrollo de las empresas. Así, podemos encontrarnos en multitud de organizaciones con planes de formación de nuevos empleados, formación continua para empleados, formación para ascensos laborales, etc.

La inversión en formación de las empresas ha sido creciente, si bien con la actual crisis económica las organizaciones han encontrado en la reducción de cursos una forma de ahorro. Las posibles consecuencias de este ahorro son la menor capacitación de los empleados y, en ocasiones, pérdida de competitividad. Debido al elevado coste empresarial que supone la formación, los gobiernos han respondido por medio de la concesión de ayudas, con el objetivo del sostenimiento de la competitividad. 
Desde los organismos públicos también se destinan fondos para la actualización y formación de los recursos humanos, en el caso español es la Fundación Tripartita para la Formación en el Empleo, la entidad estatal encargada de impulsar y coordinar la ejecución de políticas públicas en materia de Formación Profesional, en el ámbito del empleo y las relaciones laborales (atribuciones en el marco de la Ley 50/2002 de 26 de diciembre). Por otra parte, en el ámbito de la Unión Europea es el Fondo Social Europeo (FSE) (uno de los Fondos Estructurados de la Unión Europea [UE]) el encargado de las ayudas para la formación continua. El FSE se creó para reducir las diferencias económicas entre las distintas regiones y estados miembros de la UE. Por tanto, tiene por finalidad la cohesión social y económica.

Tal y como se puede observar en el cuadro 3, el presupuesto destinado a la formación de ocupados y la tasa de cobertura (entendida como el porcentaje de empresas que realizan formación para sus trabajadores respecto al total de empresas inscritas en la Tesorería General de la Seguridad Social) han sufrido un incremento significativo desde el año 2005. Esta variación, puede entenderse dentro de la toma de conciencia por parte de los gobiernos de la importancia que reúne la formación para el sostenimiento de la competitividad y en definitiva de la creación y durabilidad de la ventaja competitiva mediante la formación.

Cuadro 3: Formación Continua en España. 2005-2010

\begin{tabular}{|l|lll|}
\hline & $\begin{array}{c}\text { Presupuesto para forma- } \\
\text { ción de ocupados (millo- } \\
\text { nes de } \boldsymbol{€} \text { ) }\end{array}$ & $\begin{array}{c}\text { Empresas que han reali- } \\
\text { zado formación para sus } \\
\text { empleados }\end{array}$ & Tasa de cobertura \\
\hline $\mathbf{2 0 0 5}$ & 1.227 & 63.449 & $4,3 \%$ \\
\hline $\mathbf{2 0 0 6}$ & 1.294 & 91.161 & $5,8 \%$ \\
$\mathbf{2 0 0 7}$ & 1.303 & 136.789 & $8,4 \%$ \\
$\mathbf{2 0 0 8}$ & 1.427 & 200.689 & $12,0 \%$ \\
$\mathbf{2 0 0 9}$ & 1.506 & 293.460 & $17,8 \%$ \\
$\mathbf{2 0 1 0}$ & 1.545 & 380.548 & $24,6 \%$ \\
\hline
\end{tabular}

Fuente: Fundación Tripartita para la Formación en el Empleo. Balance de resultados 2010.

Las estadísticas de formación continua que existen de la Unión Europea, se pueden observar en el cuadro 4, referidas a los años 1999 y 2005, por no existir datos más actualizados. 
Cuadro 4: Formación Continua en las empresas Europeas, 1999 y 2005

\begin{tabular}{|c|c|c|c|c|}
\hline \multirow[b]{2}{*}{ Paises } & \multicolumn{2}{|c|}{1999} & \multicolumn{2}{|c|}{2005} \\
\hline & $\begin{array}{c}\text { \% Empresas } \\
\text { ofrecen } \\
\text { formación }\end{array}$ & $\begin{array}{l}\text { Coste de la } \\
\text { Formación }\end{array}$ & $\begin{array}{c}\text { \% Empresas } \\
\text { ofrecen } \\
\text { formación }\end{array}$ & $\begin{array}{l}\text { Coste de la } \\
\text { Formación }\end{array}$ \\
\hline Bélgica & 70 & 1,6 & 63 & 1,6 \\
\hline Bulgari & 28 & 1,0 & 29 & 1,1 \\
\hline República Checa & 69 & 1,9 & 72 & 1,9 \\
\hline Dinamarca & 96 & 3,0 & 85 & 2,7 \\
\hline Alemania & 75 & 1,5 & 69 & 1,3 \\
\hline Estonia & 63 & 1,8 & 67 & 1,6 \\
\hline Irlanda & 79 & 2,4 & 67 & 2,2 \\
\hline Grecia & 18 & 0,9 & 21 & 0,6 \\
\hline España & 36 & 1,5 & 47 & 1,2 \\
\hline Francia & 76 & 2,4 & 74 & 2,3 \\
\hline Italia & 24 & 1,7 & 32 & 1,2 \\
\hline Chipre & N.D. & N.D. & 51 & 1,3 \\
\hline Letonia & 53 & 1,1 & 36 & 0,8 \\
\hline Lituania & 43 & 0,8 & 46 & 1,2 \\
\hline Luxemburgo & 71 & 1,9 & 72 & 2,0 \\
\hline Hungría & 37 & 1,2 & 49 & 1,9 \\
\hline Malta & N.D. & N.D. & 46 & 1,8 \\
\hline Holanda & 88 & 2,8 & 75 & 2,0 \\
\hline Austria & 72 & 1,3 & 81 & 1,4 \\
\hline Polonia & 39 & 0,8 & 35 & 1,3 \\
\hline Portugal & 22 & 1,2 & 44 & 1,1 \\
\hline Rumania & 11 & 0,5 & 40 & 1,1 \\
\hline Eslovenia & 48 & 1,3 & 73 & 2,0 \\
\hline Eslovaquia & N.D. & N.D. & 60 & 1,8 \\
\hline Finlandia & 82 & 2,4 & 77 & 1,5 \\
\hline Suecia & 91 & 2,8 & 78 & 2,1 \\
\hline Reino Unido & 87 & 3,2 & 90 & 1,3 \\
\hline Noruega & 86 & 2,3 & 86 & 1,3 \\
\hline UE-27 & N.D. & N.D. & 60 & 1,6 \\
\hline UE-25 & 61 & 2,2 & 61 & 1,6 \\
\hline UE-15 & 62 & 2,3 & N.D. & N.D. \\
\hline
\end{tabular}

Coste de la Formación: \% del número de horas de formación sobre el total de horas laborables. 
N.D.: No disponible

Fuente: Eurostat. Encuesta CVTS (Continuing Vocational Training Survey in Enterprises) 2 y 3.

De la lectura de los dos cuadros 3 y 4, podemos observar como en España cada vez se destinan más fondos a la formación de los trabajadores, así como un incremento significativo del número de empresas que participan de la formación continua. El aumento de la participación, viene propiciado en ocasiones por motivos coercitivos vía leyes (p.ej. ley de prevención de riesgos laborales) o por exigencias del mercado. Los motivos de la formación dentro de la dirección estratégica se analizarán en apartados siguientes.

Aunque el incremento en España es notable desde el año 2005, y a pesar de no existir datos a nivel europeo actualizados, el porcentaje de empresas españolas que ofrecen formación está muy por debajo de la media de los países de la Unión Europea. Aunque debemos destacar que el número de horas dedicadas a la formación está próximo a la media europea.

Además de las ayudas a la formación continua vía subvenciones y bonificaciones, los gobiernos también han desarrollado ventajas fiscales a través de deducciones en el impuesto de sociedades.

Hasta hace poco, las empresas tan sólo estaban preocupadas de adquirir en el mercado laboral al trabajador con la mejor formación, ahora esto no es suficiente para mantener una ventaja respeto a la competencia. Los trabajadores deben estar formados en los últimos adelantos no sólo en un ámbito específico de la formación sino también genérico.

La búsqueda de la formación por parte de las empresas, suele surgir como respuesta a la creciente competencia del entorno externo, existiendo estudios que indican que cuando los directivos están decididos a alcanzar los objetivos estratégicos de la organización, suelen recurrir a programas de formación para buscar posibles soluciones (Sadler-Smith et al. 1998; Tsai y Tai, 2003; Wentland, 2003).

\section{LA FORMACIÓN Y LA TRANSFERENCIA DE CONOCIMIENTO}

Si consideramos que la formación es un variable que influye en el rendimiento de las empresas, la implementación de la misma debe estar vinculada a una necesidad. De lo contrario, la falta de una adecuada evaluación de las necesidades, puede dificultar la eficacia de los programas de formación y ser aplicados de forma incorrecta como solución a algún problema de rendimiento (Semler, 1997; Sadler-Smith, Sargeant y Dawson, 1998; Montesino, 2002; Tannebaum, 2002; Noe, 2005; Chi, Wu y Lin, 2008; Eerde et al., 2008).

En aquellas empresas que no realizan una correcta valoración de las necesidades de formación de sus empleados, la probabilidad de no retorno de la inversión producida es mayor. En ocasiones, la formación en organizaciones responde a presiones 
institucionales que el entorno ejerce sobre la empresa. De modo que la formación no es considerada por la organización como fuente de ventaja competitiva sostenible, sino como mejora de legitimidad.

La empresa está sometida a unas presiones, aunque debe valorar la formación que necesita impartir a sus empleados, al margen de la obtención de la legitimidad social.

En la actualidad el trabajo se ha convertido cada vez más en adquisición de conocimientos, la inversión en activos intangibles se ha incluido dentro de la estrategia empresarial para la adquisición de ventajas competitivas y las empresas precisan que los conocimientos adquiridos por los trabajadores se transfieran a su actuación diaria.

A través del meta-análisis llevado a cabo por Blume et al. (2010), consideramos que la incidencia de la formación en los resultados empresariales puede estar influida por la transferencia de los conocimientos. Las organizaciones empresariales no sólo han de ser conscientes de las necesidades de formación que poseen sus empleados y en especial sus directivos, sino que además éstos una vez que han recibido dicha formación deben ser capaces de ponerlos en práctica. Es necesario que el aprendizaje obtenido se transfiera al trabajo y produzca cambios significativos en el rendimiento (Goldstein y Ford, 2002; Blume et al., 2010), esto es lo que se conoce como transferencia positiva de la formación. Las empresas desean que toda la inversión en formación suponga un incremento del rendimiento, pero existen problemas o factores que influyen en la transferencia y por tanto en el retorno de la inversión en la organización.

El concepto de transferencia del conocimiento ha sido durante bastantes años un término más próximo a la rama de la psicología y la educación (Grose y Briney, 1963; Barnett y Ceci, 2002). Originalmente, se define como la medida en que el aprendizaje de una tarea o situación influye en otras tareas o situaciones (Adams, 1987). Las investigaciones posteriores, han permitido concluir que la transferencia del conocimiento, es más probable que exista en aquellas tareas que son muy similares a las tareas aprendidas y menos en las que las tareas y situaciones de aprendizaje son muy diferentes a las aprendidas (Blume et al., 2010). Siguiendo la investigación que llevamos a cabo, consideramos más apropiada la definición de transferencia utilizada por Blume et al. (2010) como "conjunto de dos dimensiones principales: generalización (medida en que los conocimientos y habilidades adquiridos en un entorno de aprendizaje se aplican a diferentes entornos, personas y/o situaciones de las personas capacitadas) y mantenimiento (medida en que los cambios que resultan de una experiencia de aprendizaje persisten en el tiempo)". Por tanto, para poder analizar el efecto de la formación sobre los resultados, es necesaria la adecuación a una necesidad y la transferencia positiva de ese conocimiento.

Una de las lagunas existentes en el análisis de la transferencia de conocimientos, es el estudio de cómo afectan las habilidades cerradas y abiertas. Se entiende por habilidades cerradas a aquellas vinculadas al aprendizaje de habilidades específicas que se van a producir de forma idéntica en el entorno de la transferencia como en el contexto de aprendizaje. Las abiertas son las vinculadas a los principios de aprendizaje. Con las habilidades cerradas, se tiene la oportunidad de aplicar las habilidades 
aprendidas de inmediato en el trabajo. Las oportunidades para aplicar las habilidades abiertas son menos evidentes y puede precisar de la colaboración o supervisión. De modo, que el apoyo de un supervisor en la transferencia, puede ser diferente según se trate de habilidades abiertas o cerradas. En las abiertas, se tienen más posibilidades de elección respecto a la transferencia. Los trabajadores motivados para aprender habilidades abiertas buscan más oportunidades en el lugar de trabajo para aplicar la formación recibida y también buscan un compañero de trabajo de apoyo a la aplicación de las habilidades entrenadas (Blume et al., 2010).

Numerosos estudios han analizado la importancia de la formación para la creación y sostenibilidad en el tiempo de una ventaja competitiva. Los principales estudios que han incluido la formación como práctica "excelente" se pueden ver en el cuadro 6 .

\section{CONCLUSIONES}

El presente trabajo ha analizado numerosos estudios que muestran que la obtención de éxito por parte de las organizaciones, se debe en buena parte a la capacidad para generar y mantener en el tiempo una ventaja competitiva.

La teoría de recursos y capacidades señala que la obtención de una ventaja competitiva, depende no tanto del sector específico en el que se desarrolle el negocio; sino de los recursos y capacidades diferenciadores de una empresa.

Evidentemente, no todos los recursos son capaces de generar ventajas competitivas, sino solo aquellos que son considerados estratégicos. Es decir, los recursos escasos, valiosos, limitadamente sustituibles e imperfectamente imitables.

Los recursos humanos intangibles asociados a las personas, y englobados bajo la denominación de capital humano, suelen cumplir las condiciones necesarias, para obtener una ventaja competitiva sostenible en el tiempo, capaz de generar rentas duraderas. Por este motivo, el capital humano se ha convertido una de las claves sobre las que se basa el éxito de la organización.

La generación y acumulación de capital humano, puede obtenerse por dos vías: el aprendizaje y la experiencia (Danvila y Sastre, 2009). Dado que la experiencia se obtiene con el devenir del tiempo, el aprendizaje resulta ser un elemento crucial para la generación de capital humano.

El aprendizaje puede lograrse en mayor cuantía mediante una adecuada política de formación de personal.

De esta forma, la formación se convierte en un elemento clave para la obtención de ventajas competitivas sostenibles en el tiempo, capaces de generar rentas duraderas. 
Cuadro 5: Estudios empíricos sobre la formación

\begin{tabular}{|c|c|c|c|}
\hline \multirow[t]{2}{*}{ FORMACIÓN } & AUTOR & \multicolumn{2}{|c|}{ RESULTADOS } \\
\hline & Russell et al. (1985) & 4 & Imagen de la firma \\
\hline \multirow{4}{*}{$\begin{array}{l}\text { Énfasis en la forma- } \\
\text { ción }\end{array}$} & & 4 & Productividad \\
\hline & Arthur (1994) & 4 & Calidad \\
\hline & & 4 & Ratios de trabajadores \\
\hline & Delery and Doty (1996) & $\oplus$ & Rentabilidad \\
\hline Formación inicial & Bartel (1994) & 4 & Productividad \\
\hline \multirow{4}{*}{$\begin{array}{l}\text { Horas de forma- } \\
\text { ción/empleado }\end{array}$} & Kidder and Rouiller (1997) & 4 & Productividad \\
\hline & & 4 & Calidad \\
\hline & Danvila and Sastre (2009) & 4 & Rentabilidad \\
\hline & Bishop (1994) & 4 & Productividad \\
\hline \multirow{2}{*}{$\begin{array}{l}\text { Número de clases } \\
\text { recibidas }\end{array}$} & Huselid (1995) & $\oplus$ & Productividad \\
\hline & Russell et al. (1985) & $\oplus$ & Imagen de la firma \\
\hline \multirow{4}{*}{$\begin{array}{l}\text { Número de empleados } \\
\text { formados }\end{array}$} & Barrett and O'Connell (2001) & 4 & Productividad \\
\hline & Russell et al. (1985) & 4 & Productividad \\
\hline & Danvila et al. (2009) & 4 & Productividad \\
\hline & Delaney and Huselid (1996) & 4 & Imagen de la firma \\
\hline \multirow{3}{*}{$\begin{array}{l}\text { Porcentaje de emplea- } \\
\text { dos formados }\end{array}$} & Blanch and Lynch (1996) & $\oplus$ & Productividad \\
\hline & Ichniowski et al. (1997) & $\varphi$ & Productividad \\
\hline & Murray and Raffaele (1997) & $\oplus$ & Calidad \\
\hline \multirow{2}{*}{ Alta/baja formación } & Barrett and O'Connell (2001) & 4 & Productividad \\
\hline & Bartel (1994) & 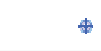 & Productividad \\
\hline \multirow{3}{*}{ Costes directos } & Murray and Raffaele (1997) & 4 & Calidad \\
\hline & D’Arcimoles (1997) & $\oplus$ & Q de Tobin \\
\hline & Bishop (1994) & 4 & Productividad \\
\hline \multirow{6}{*}{$\begin{array}{l}\text { Tipos de actividades } \\
\text { formativas }\end{array}$} & Danvila and Sastre (2009) & $\varphi$ & Productividad \\
\hline & D’Arcimoles (1997) & & \\
\hline & Barrett and O'Connell (2001) & & \\
\hline & Danvila et al. (2009) & 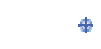 & Productividad \\
\hline & Barron and Berger (1999) & & \\
\hline & Bartel (1994) & & \\
\hline
\end{tabular}


Cuadro 5: Estudios empíricos sobre la formación (cont.)

\begin{tabular}{|c|c|c|c|}
\hline \multirow{4}{*}{$\begin{array}{l}\text { Formación / No } \\
\text { formación }\end{array}$} & Delaney and Huselid (1996) & 4 & Imagen de la firma \\
\hline & & 4 & Participación \\
\hline & & 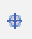 & Indicadores de RR.HH. \\
\hline & & 4 & Calidad \\
\hline \multirow{4}{*}{$\begin{array}{l}\text { Métodos, característi- } \\
\text { cas y actividades de } \\
\text { formación }\end{array}$} & Aragón et al. (2003) & $\oplus$ & Productividad \\
\hline & & 4 & Ventas \\
\hline & & 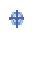 & $\begin{array}{l}\text { Beneficios antes de intereses e } \\
\text { impuestos }\end{array}$ \\
\hline & & $\rightarrow$ & Rentabilidad de las ventas \\
\hline
\end{tabular}

Fuente. Elaboración propia a partir de Úbeda García (2005: 1697).

A pesar de la crisis económica el esfuerzo inversor por empleado ha crecido en los últimos años, si bien es cierto que debido en gran parte a ayudas y subvenciones, muchas de ellas obtenidas a través del Fondo Social Europeo.

En la actualidad, la formación genera capital humano y es una herramienta clave para la transferencia de conocimiento. Por este motivo, la formación es una pieza clave y produce efectos directos y reales sobre los resultados empresariales.

Numerosos estudios empíricos han demostrado que las empresas que realizan un mayor esfuerzo en formación suelen obtener rentas económicas superiores a los de sus competidores.

Estos estudios convierten a la formación en un aspecto de primer orden, en los estudios empíricos que buscan las causas últimas del éxito empresarial. De esta forma se comprende la importancia de la formación en las empresas y los abundantes estudios empíricos que analizan esta relación y sus efectos.

\section{BIBLIOGRAFÍA}

ABDEL-WAHAB, M., DAINTY, A., ISON, S. y HAZLEHURST, G. An exploration of the relationship between training grants and profitability of UK construction companies. International Journal of Training \& Development, Vol. $12, \mathrm{n}^{\circ} .3,2008$, p. 188.

ADAMS, J.A. Historical Review and Appraisal of Research on the Learning, Retention, and Transfer of Human Motor Skills. Psychological bulletin, Vol. 101, $\mathrm{n}^{\mathrm{o}}$. 1, 1987, p. 41.

ALAVI, M. y LEIDNER, D.E. Review: Knowledge management and knowledge management systems: Conceptual foundations and research issues. MIS Quarterly, Vol. 25, nº 1, 2001, p. 107. 
AMIT, R. y SCHOEMAKER, P.J.H. Strategic assets and organizational rent. Strategic Management Journal, Vol. 14, nº 1, 1993, p. 33.

ANSOFF, H.I. Corporate strategy, New York: McGraw-Hill, 1965. 241 p. ISBN: 978-0070021112.

ARAGÓN, A., BARBA, M.I. y SANZ, R. Effects of training in business perfomance. International Journal of Human Resource Management, Vol. 14, $\mathrm{n}^{\mathrm{o}}$. 6, 2003, p. 956-980.

ARTHUR, J.B. Effects of human resource systems on manufacturing performance and turnover. Academy of Management Journal, Vol. 37, nº 3, 1994, p. 670-670.

BACON, N. y HOQUE, K. HRM in the SME sector: valuable employees and coercive networks. The International Journal of Human Resource Management, Vol. $16, \mathrm{n}^{\mathrm{o}} .11,2005$, p. 1976.

BARNETT, S.M. y CECI, S.J. When and Where do we apply what we learn? A taxonomy for far transfer. Psychological Bulletin, Vol. 128, $n^{\circ} .4$, 2002, p. 612-637.

BARNEY, J.B. Integrating Organizational Behavior and Strategy Formulation Research: A Resource Based Analysis. En SHRIVASTIVA, P., HUFF, A. y DUTTON, J. Advances in Strategic Management, Londres: JAI Press, 2002, p. 39-62. ISBN: I-55938-377-I.

BARNEY, J.B. Special theory forum. The resource-based model of the firm: Origins, implications and prospects. Journal of Management, Vol. 17, $\mathrm{n}^{\mathrm{o}} .1,1991, \mathrm{p}$. 97-118.

BARNEY, J.B. y WRIGHT, P.M. On becoming a strategic partner: The role of human resources in gaining competitive advantage, Human resource management, Vol. 37, no. 1, 1998, p. 31.

BARRETT, A. y O'CONNELL, P.J. Does training generally work? The returns to incompany training. Industrial \& labor relations review, Vol. 54, $\mathrm{n}^{\circ} .3,2001$, p. 647.

BARRON, J.M., BERGER, M.C. y BLACK, D.A. Do workers pay for on-the-job training?. The Journal of human resources, Vol. 34, no. 2, 1999, p. 235.

BARTEL, A.P. Productivity gains from the implementation of employee training programs. Industrial Relations, Vol. 33, nº . 4, 1994, p. 411.

BASSI, L.J., CHENEY, S. y MCMURRER, D. A common standard for measuring training results. Training \& Development, Vol. 52, nº 3, 1998, p. 10.

BATT, R. Managing customer services: Human resource practices, quit rates, and sales growth. Academy of Management Journal, Vol. 45, no. 3, 2002, p. 587.

BEAVER, G. y HUTCHINGS, K. Training and developing an age diverse workforce in SMEs: The need for a strategic approach. Education \& Training, $\mathrm{n}^{\circ} .47,2005$, p. $592-604$.

BECKER, G.S. El capital humano, Madrid: Alianza, 1983. 288 p. ISBN: 9788420680637. 
BLACK, J.A. Y BOAL, K.B. Strategic resources: Traits, configurations and paths to sustainable competitive advantage. Strategic Management Journal, $\mathrm{n}^{\circ}$. 15, 1994, p. 131.

BLACK, S.E. y LYNCH, L.M. Human-capital investments and productivity. The American Economic Review, Vol. 86, nº. 2, 1996, p. 263.

BLUME, B.D., FORD, J.K., BALDWIN, T.T. y HUANG, J.L. Transfer of Training: A Meta-Analytic Review. Journal of Management, Vol. 36, nº. 4, 2010, p. 1065.

BOXALL, P. The strategic HRM debate and the resource-based view of the firm. Human Resource Management Journal, Vol. 6, nº 3, 1996, p. 59.

BRYAN, J. Training and Performance in Small Firms. International Small Business Journal, Vol. 24, nº 6, 2006, p. 635.

CAVES, R.E. Industrial organization, corporate strategy, and structure. Journal of Economic Literature, Vol. 18, $n^{\circ} .1,1980$, p. 64-92.

CHI, N., WU, C. y LIN, C. Does training facilitate SME's performance?. The International Journal of Human Resource Management, Vol. 19, nº 10, 2008, p. 1962.

DACIN, M.T., OLIVER, C. y ROY, J. The legitimacy of strategic alliances: an institutional perspective. Strategic Management Journal, Vol. 28, nº. 2, 2007, p. 169.

DANVILA DEL VALLE, I. y SASTRE CASTILLO, M.A. The generation of human capital through training as a source of sustainable competitive advantage. Esic Market. Economic and Business Journal, $n^{\circ}$. 128, 2007, p. 109-144.

DANVILA DEL VALLE, I. y SASTRE CASTILLO, M.A. Human capital and sustainable competitive advantage: an analysis of the relationship between training and performance. The International Entrepreneurship and Management Journal, Vol. 5, no. 2, 2009, p. 139-163.

DANVILA DEL VALLE, I., SASTRE CASTILLO, M.A. y RODRÍGUEZ DUARTE, A. The effects of training on performance in service companies: a data panel study. International Journal of Manpower, Vol. 30, n. ${ }^{\circ} 4$, 2009, p. 393-407.

DE CASTRO, J.O. y CHRISMAN, J.J. Order of market entry, competitive strategy, and financial performance. Journal of Business Research, Vol. 33, $\mathrm{n}^{\circ} .2,1995$, p. 165.

DE KOK, J. The impact of firm-provided training on production. International Small Business Journal, Vol. 20, no. 3, 2002, p. 271.

DE LA ROSA, $M^{\mathrm{a}}$. D. y CARMONA, A. Cómo afecta la relación del empleado con el líder a su compromiso con la organización. Universia Business Review, $\mathrm{n}^{\circ}$. Segundo Trimestre, 2010, p. 112-132.

DE SAÁ, P. y GARCÍA, J.M. A resource-based view of human resource management and organizational capabilities development. International Journal of Human Resource Management, Vol. 13, $\mathrm{n}^{\circ} .1,2002$, p. 123-140.

DELANEY, J.T. y HUSELID, M.A. The impact of human resource management practices on perceptions of organizational performance. Academy of Management Journal, Vol. 39, nº 4, 1996, p. 949-949. 
DELERY, J.E. y DOTY, D.H. Modes of theorizing in strategic human resource management: Tests of universalistic, contingency, and configurational performance predictions. Academy of Management Journal, Vol. 39, nº 4, 1996, pp. 802.

DIERICKX, I., COOL, K. y BARNEY, J.B. Asset Stock Accumulation And Sustainability Of Competitive. Management Science, Vol. 35, no . 12, 1989, p. 1504.

DIMAGGIO, P.J. y POWELL, W.W. The iron cage revisited: Institutional isomorphism and collective rationality in organizational fields. American Sociological Review, $n^{\circ} .48,1983$, p. 147-160.

FEY, C.F. y BJORKMAN, I. The effect of human resource management practices on MNC subsidiary performance in Russia. Journal of international business studies, Vol. 32, nº 1, 2001, p. 59.

GOLDSTEIN, I. y FORD, J.K. Training in organizations. Cuarta edición. Belmont, CA: Wadsworth, 2002. 432 p. ISBN: 9780534345549.

GRANT, R.M. Prospering in dynamically-competitive environments: Organizational capability as knowledge integration. Organization Science, Vol. 7, $\mathrm{n}^{\circ} .4,1996, \mathrm{p}$. 375.

GRANT, R.M. The Resource-Based Theory of Competitive Advantage: Implications for Strategy Formulation. California Management Review, Vol. 33, nº. 3, 1991, p. 114.

GROSE, R.F. y BRINEY, R.C. Transfer of learning: An enduring problem in psychology., Princeton, NJ: Van Nostrand, 1963. 194 p.

GUEST, D.H. Human resource management and perfomance: A review and research agenda. International Journal of Human Resource Management, $\mathrm{n}^{\circ} .8$, 1997, p. 263-276.

HAYES, R.H., WHEELWRIGHT, S.C. y CLARK, K.B. Dynamic manufacturing. Nueva York: The Free Press, 1988. 429 p. ISBN: 0-02-914211-3.

HUSELID, M.A. The impact of human resource management practices on turnover, productivity, and corporate financial performance. Academy of Management Journal, Vol. 38, no. 3, 1995, p. 635.

ICHNIOWSKI, C., SHAW, K. y PRENNUSHI, G. The effects of human resource management practices on productivity: A study of steel finishing lines. The American Economic Review, Vol. 87, nº. 3, 1997, p. 291-313.

KAMOCHE, K. Strategic human resource management within a resource-capability view of the firm. The Journal of Management Studies, Vol. 33, nº. 2, 1996, p. 213.

KLEIN, H.J. y WEAVER, N.A. The effectiveness of an organizational-level orientation training program in the socialization of new hires. Personnel Psychology, Vol. 53, no. 1, 2000, p. 47.

LADO, A.A. y WILSON, M.C. Human resource systems and sustained competitive advantage: A competency-based perspective. Academy of Management Review, Vol. 19, nº. 4, 1994, p. 699. 
LIN, C.Y. y WEI, Y. An Eastern and SME version of expatriate management: an empirical study of small and medium enterprises in Taiwan. The International Journal of Human Resource Management, Vol. 16, $\mathrm{n}^{\circ} .8$, 1995, p. 1431.

MAKÓ, C. Training and Competence Development in the SME Sector: The Hungarian Case. Journal for East European Management Studies, Vol. 10, $n^{\circ} .2,2005$, p. 156.

MEYER, J.W. y ROWAN, B. Institutionalized organizations: Formal structure as myth as ceremony. American Journal of Sociology, $n^{\circ} .83,1977$, p. 340-363.

MUELLER, F. Human resources as strategic assets: An evolutionary resource-based theory. The Journal of Management Studies, Vol. 33, no. 6, 1996, p. 757.

NELSON, R.R. y WINTER, S.G. An evolutionary theory of economic change, Cambridge: Belknap Press, 1982. 437 p. ISBN: 9780674272286.

NG, Y.C. y SIU, N.Y.M. Training and enterprise performance in transition: evidence from China. The International Journal of Human Resource Management, Vol. 15, $n^{\circ} .4 / 5,2004$, p. 878.

PATTON, D., MARLOW, S. y HANNON, P. The relationship between training and small firm performance; research frameworks and lost quests. International Small Business Journal, Vol. 19, no. 1, 2000, p. 11.

PENROSE, E.T. The theory of growth of the firm. London: Basil Blackwell, 1959. 249 p. ISBN: 978-0198289777.

PETERAF, M.A. The Cornerstones of Competitive Advantage: a Resource-Based View. Strategic Management Journal (1986-1998), Vol. 14, nº. 3, 1993, p. 179.

PFEFFER, J. Competitive advantage through people. California Management Review, Vol. 36, no. 2, 1994, p. 9.

ROTHENBERG, S. Environmental managers as institutional entrepreneurs: The influence of institutional and technical pressures on waste management. Journal of Business Research, Vol. 60, nº 7, 2007, p. 749.

RUMELT, R.P. How Much Does Industry Matter?. Strategic Management Journal, Vol. 12, no. 3, 1991, p. 167.

RUMELT, R.P. Toward a strategic theory of the firm in competitive strategic management. En LAMB, R.B. Competitive strategic management. Englewood Cliffs, New Jersey: Prentice Hall, 1984, pp. 556-570. ISBN: 9780131549722.

RUSSELL, J., TERBORG, J. y POWERS, M. Organizational Productivity and Organizational Level Training and Support. Personnel Psychology, $n^{\circ} .38,1985, p$. 849-863.

SADLER-SMITH, E., SARGEANT, A. y DAWSON, A. Higher level skills training and SMEs. International Small Business Journal, Vol. 16, $\mathrm{n}^{\circ} .2$ 2, 1998, p. 84.

SCOTT, W.R. Institutions and organizations. Segunda edición. Thousand Oaks, CA: Sage,.2001. 280 p. ISBN: 9780761920007.

SEMLER, S.W. Systematic agreement: A theory of organizational alignment. Human Resource Development Quarterly, Vol. 8, nº 1, 1997, p. 23. 
STAVROU, E., BREWSTER, C. y CHARALAMBOUS, C. Human resource management and firm performance in Europe through the lens of business systems: best fit, best practice or both?. The International Journal of Human Resource Management, Vol. 21, n'. 7, 2010, p. 933-962.

STOREY, D.J. Exploring the link, among small firms, between management training and firm performance: A comparison between the UK and other OECD countries. International Journal of Human Resource Management, $n^{\circ} .15,2004, p .112$.

TEECE, D.J., PISANO, G. y SHUEN, A. Dynamic capabilities and strategic management. Strategic Management Journal, Vol. 18, no. 7, 1997, p. 509.

TSAI, W. y TAI, W. Perceived importance as a mediator of the relationship between training assignment and training motivation. Personnel Review, Vol. 32, $\mathrm{n}^{\mathrm{o}} 1 / 2$, 2003, p. 151.

ÚBEDA GARCÍA, M. Training and business performance: the Spanish case. The International Journal of Human Resource Management, Vol. 16, $\mathrm{n}^{\mathrm{o}} .9,2005$, $\mathrm{p}$. 1691.

VAN DE WIELE, P. The impact of training participation and training costs on firm productivity in Belgium. The International Journal of Human Resource Management, Vol. 21, no 4, 2010, p. 582-599.

WENTLAND, D. The strategic training of employees model: Balancing organizational constraints and training content. Advanced Management Journal, Vol. 68, $\mathrm{n}^{\circ} .1,2003$, p. 56.

WERNERFELT, B. A Resource-Based View of the Firm. Strategic Management Journal, Vol. 5, nº. 2, 1984, p. 171.

WILLIAMSON, O.E. The economics institutions of capitalism: Firm, markets, and relations contracting. Nueva York: Free Press, 1985. 450 p.

WILLIAMSON, O.E. 1975, Markets and hierarchies. Nueva York: Free Press, 1975. $286 \mathrm{p}$.

WRIGHT, P.M., MCMAHAN, G.C., MCCORMICK, B. y McWILLIAMS, A. Strategy, Core Competence, and $\mathrm{Hr}$ Involvement as Determinants of $\mathrm{Hr}$ Effectiveness and Refinery Performance Human Resource Management (1986-1998), Vol. 37, $\mathrm{n}^{\mathrm{o}} .1,1998$, p. 17.

WRIGHT, P.M., MCMAHAN, G.C., y McWILLIAMS, A.. Human resources and sustained competitive advantage: A resource-based view perspective. International Journal of Human Resource Management, $n^{\circ} .5$, p. 301-326. 